(1)

\title{
Perfil clínico e uso de serviços de saúde em idosos
}

\author{
Pricila C. C. Ribeiro, ${ }^{1 \star}$ Eliane F. C. Banhato, ${ }^{2}$ Danielle V. Guedes ${ }^{3}$
}

\section{Resumo}

Introdução: Conhecer o perfil clínico-funcional e os fatores associados ao uso de serviços de saúde entre idosos pode auxiliar profissionais no desenvolvimento de ações preventivas e de cuidado voltadas para esta população. Objetivo: Descrever o perfil clínico de idosos da comunidade, apresentando as prevalências das doenças crônicas e agravos à saúde. Verificou-se também a associação das condições patológicas com o uso de serviços de saúde e com características sociodemográficas. Materiais e métodos: Inquérito realizado no Estudo FIBRA-JF, conduzido com 426 idosos (>65 anos) do município de Juiz de Fora-MG. Os dados sociodemográficos, de diagnósticos das doenças crônicas, de agravos e de uso de serviços de saúde foram obtidos por autorrelato. Resultados: O perfil clínico da amostra apresentou idosos sem grandes prejuízos funcionais, sendo a hipertensão arterial a patologia mais prevalente (37,9\%). Cardiopatias ( $\mathrm{p}=0,072$ ) e câncer $(\mathrm{p}=0,013)$ foram mais prevalentes entre os homens, e entre as mulheres, artrite reumatoide $(p=0,002)$, depressão $(p=0,040)$, incontinência urinária $(\mathrm{p}=0,037)$ e osteoporose $(\mathrm{p}<0,001)$. A prevalência de fraturas por quedas foi mais alta para o grupo com 85 anos ou mais, comparado às demais faixas etárias $(\mathrm{p}=0,003)$. Foi alta $\mathrm{a}$ frequência de idosos com acesso a plano de saúde $(60,2 \%)$, o que esteve associado a um maior número de consultas médicas ( $\mathrm{p}=0,01)$. Conclusões: Os resultados foram compatíveis com o que vem sendo relatado na literatura. $O$ acesso a plano de saúde foi mais amplo, comparado à população idosa geral no país, o que pode explicar o uso mais frequente de serviços de saúde como consultas e internações hospitalares.

Descritores: Perfil de saúde; Serviços de saúde; Envelhecimento.

\section{Abstract}

\section{Clinical profile and health service use in the elderly}

Introduction: Information on medical and functional profiles and other factors associated with healthcare services use by the elderly can be helpful in planning prevention and direct care actions for this population. Objectives: To describe the community-dwelling elderly's clinical profile, presenting the prevalence of chronic diseases and health problems. A correlation between pathology conditions, health services use, and sociodemographic characteristics was also verified. Methods: Research done in the FIBRA-JF Study, conducted with 426 elderly ( $\geq 65$ years) from Juiz de Fora, MG, Brazil. Sociodemographic, chronic disease, health conditions, and health services use data were obtained by self-report. Results: The clinical sample presented elderly without major functional loss, with arterial hypertension being the most common

\author{
1. Departamento de Psicologia. Faculdade de Filosofia e Ciências \\ Humanas. Universidade Federal de Minas Gerais. Belo Horizonte, \\ MG, Brasil. \\ 2. Departamento de Psicologia. Centro de Ensino Superior de Juiz de \\ Fora. Juiz de Fora, MG, Brasil. \\ 3. Departamento de Psicologia. Universidade Federal de Juiz de Fora. \\ Juiz de Fora, MG, Brasil.
}

\section{*Endereço para correspondência:}

Departamento de Psicologia, FFCH, UFMG

Avenida Antônio Carlos, 6.627

Belo Horizonte, MG, Brasil. CEP: 31270-901.

E-mail: pricilaribeiro@ufmg.br

Revista HUPE, Rio de Janeiro, 2018;17(2):25-34

Recebido em 07/05/2018. Aprovado em 23/07/2018.

disease (37.9\%). Heart diseases $(\mathrm{p}=0.072)$ and cancer $(\mathrm{p}=0.013)$ were prevalent in men, while rheumatoid arthritis $(\mathrm{p}=0.002)$, depression $(\mathrm{p}=0.040)$, urinary incontinence $(\mathrm{p}=0.037)$, and osteoporosis $(\mathrm{p}<0.001)$ were prevalent in women. Bone fractures due to falls were more common in the 85 years old or more group, compared to the other groups ( $\mathrm{p}=0.003$ ). Private health insurance coverage access was high $(60.2 \%)$, which was associated with more medical appointments $(\mathrm{p}=0.01)$. Conclusions: The results were compatible with reported in literature. Private health insurance access was broader, compared to the average of the elderly population in Brazil, which may explain the freuquent use of health services such as medical appointments and hospitalizations..

Keywords: Health; Health services; Aging.

\section{Resumen}

Perfil clínico y uso de servicios de salud en ancianos

Introducción: Conocer el perfil clínico-funcional y los factores asociados al uso de servicios de salud entre ancianos puede auxiliar profesionales en el desarrollo de acciones preventivas y de cuidado dirigidas a esta población. Objetivo: Describir el perfil clínico de ancianos comunitarios, presentando la prevalencia de las enfermedades crónicas y agravios a la salud. Se verificó también la asociación de las condiciones patológicas con el uso de servicios de salud y con características sociodemográficas. Materiales y métodos: Encuesta realizada en el Estudio FIBRA-JF, conducido con 426 ancianos ( $>65$ años) del municipio de Juiz de Fora-MG. Los datos sociodemográficos, de diagnósticos de las enfermedades crónicas, de agravios y 


\section{Artigo original}

de uso de servicios de salud fueron obtenidos por autorrelato. Resultados: El perfil clínico de la muestra presentó ancianos sin grandes pérdidas funcionales, siendo la hipertensión arterial la patología más prevalente (37,9\%). Enfermedades del corazón $(\mathrm{p}=0,072)$ y cáncer $(\mathrm{p}=0,013)$ fueron más prevalentes entre los hombres, mientras que en las mujeres, la artritis reumatoide ( $\mathrm{p}=0,002$ ), la depresión ( $\mathrm{p}=0,040$ ), la incontinencia urinaria $(\mathrm{p}=0,037)$ y la osteoporosis $(\mathrm{p}<0,001)$. La prevalencia de fracturas por caídas fue más elevadas en el grupo de 85 años o más, comparado a las demás edades ( $\mathrm{p}=0,003)$. La frecuencia de ancianos con acceso a un plan de salud fue alta (60,2\%), lo que estuvo asociado al mayor número de consultas médicas ( $\mathrm{p}=0,01)$. Conclusiones: Los resultados fueron compatibles con lo que viene siendo relatado en la literatura. El acceso a un plan de salud fue más amplio, comparado a la población anciana general en el país, lo que puede explicar el uso de servicios de salud como consultas e internaciones hospitalarias..

Palabras clave: Perfil de salud; Servicios de salud; Envejecimiento.

sobre a saúde de indivíduos idosos também vem sendo demonstrado a partir da associação da menor renda e da baixa escolaridade, com a maior prevalência de doenças crônicas. ${ }^{10,11}$ Esses dois fatores também estão correlacionados a um maior risco para mortalidade. ${ }^{12}$

Para mudar o cenário desfavorável de aumento da prevalência de agravos crônicos de saúde, a Organização das Nações Unidas iniciou debates sobre o tema e, na atualidade, diversos países seguem um plano estratégico para redução da mortalidade por doenças crônicas não transmissíveis (DCNT). ${ }^{13}$ Acompanhando este movimento, foi adotado no Brasil o 'Plano de Ações Estratégicas para o Enfrentamento das DCNT no Brasil, 2011-2022', no qual podem ser encontradas as metas e os compromissos, bem como as ações e prioridades, para enfrentamento das DCNT e de seus fatores de risco no país. ${ }^{14}$

Neste contexto, investigações populacionais que visam descrever o perfil de adoecimento e de cuidados em saúde da população mais velha podem contribuir para o dimensionamento da demanda real dos idosos por serviços de saúde, de forma a auxiliar os gestores no desenvolvimento de ações preventivas e de assistência. Sendo assim, o objetivo deste estudo foi descrever o perfil clínico dos idosos no estudo FIBRAJF, em Minas Gerais, apresentando as prevalências das doenças crônicas e agravos à saúde. Além disto, verificou-se a associação das condições patológicas com o uso de serviços de saúde e com as características sociodemográficas desta população.

\section{Materiais e métodos}

Esta pesquisa foi parte do projeto multicêntrico rede FIBRA Brasil, de delineamento transversal e analítico, conduzido pelo polo UERJ, realizado com os dados da população idosa do município de Juiz de 
Fora. ${ }^{15}$ A pesquisa respeitou todos os padrões éticos aprovados pela Comissão Nacional deÉtica em Pesquisa do Ministério da Saúde (CONEPE/MS - 313/2008) sob o processo número 555087/2006-9 com o apoio do Conselho Nacional de Desenvolvimento Científico e Tecnológico (CNPq). A coleta de dados ocorreu nos anos 2008-2009, nas residências dos idosos, e foi realizada por pesquisadores da Universidade Federal de Juiz de Fora devidamente treinados. ${ }^{16}$

Os dados do questionário FIBRA-JF analisados no presente estudo foram:

1) Características socioeconômicas e demográficas: sexo, idade, renda, escolaridade e situação conjugal.

2) O autorrelato de doenças foi obtido com a seguinte pergunta: "No último ano, algum médico já disse que o(a) senhor(a) tem os seguintes problemas de saúde?": doença do coração como angina, infarto do miocárdio ou ataque cardíaco; pressão alta - hipertensão; derrame/AVC/Isquemia; diabetes Mellitus; tumor maligno/câncer; artrite ou reumatismo; doença do pulmão (bronquite e enfisema); depressão; osteoporose.

3) O autorrelato de agravos à saúde foi obtido com a seguinte pergunta: "Nos últimos 12 meses o(a) Sr(a) teve algum desses problemas?": Incontinência urinária (ou perda involuntária da urina)?; Incontinência fecal (ou perda involuntária das fezes)?. Além disto, foi avaliada a ocorrência de fraturas com a seguinte pergunta: "Devido às quedas, o(a) senhor(a) sofreu alguma fratura?"; tabagismo, com a pergunta: "O (a) $\mathrm{Sr}$ (a) fuma atualmente?"; e acuidade visual e auditiva com as perguntas: "O(a) senhor(a) ouve bem?"; “O(a) senhor(a) usa aparelho auditivo?"; "O(a) senhor enxerga bem?" e "O(a) senhor(a) usa óculos ou lentes de contato?".

4) A capacidade funcional foi mensurada com a escala de Katz que verifica o grau de dependência para realização de atividades básicas da vida diária - ABVD - (tomar banho, vestir-se, higiene pessoal, alimentar-se, continência esfincteriana e transferir-se de um local para outro) e a escala de Lawton, baseado no índice de Lawton e Brody, que avalia a capacidade para realizar atividades instrumentais da vida diária - AIVD - (usar o telefone, fazer compras, sair de casa sozinho, preparar refeições, realizar tarefas domésticas, gerenciar finanças e tomar medicamentos). ${ }^{17-20} \mathrm{O}$ resultado destas escalas permite a descrição da funcionalidade, variando de independência completa até a dependência total para realização das atividades de vida diária.

5) O uso e o acesso aos serviços de saúde foram avaliados com as seguintes perguntas: a) “O(A) Sr.(a) tem plano de saúde?"; b) "Precisou ser internado(a) no hospital pelo menos por 1 noite?"; c) $O(A)$ senhor(a) recebeu em sua casa a visita de algum profissional da área da saúde? (enfermeiro, médico, fisioterapeuta, psicólogo, fonoaudiólogo); d) Quantas vezes o(a) senhor(a) foi à uma consulta médica (qualquer especialidade)? e (para aqueles que responderam nenhuma) "Qual o principal motivo de não ter ido ao médico nos últimos 12 meses?"; e) "Quantos medicamentos o(a) senhor(a) tem usado de forma regular nos últimos 3 meses, receitados pelo médico ou por conta própria?; e, para os que tomam medicamentos, "Como tem acesso aos medicamentos"? e "O(A) senhor(a) deixa de tomar algum medicamento prescrito por dificuldade financeira para comprá-lo?".

\section{Análise dos dados}

Foram calculadas as prevalências das doenças e agravos à saúde relatados pela população total, segundo sexo e grupo etário. Foram realizadas análises com teste $X^{2}$ de Pearson para verificar a associação do acesso e uso de serviços de saúde com as patologias e os agravos da saúde e com as características sociodemográficas. Foi realizado teste $t$ de Student para comparar as médias de consultas médicas realizadas entre os grupos de participantes acometidos e não acometidos pelas patologias investigadas.

Para verificar a hipótese de que o aumento da quantidade de consultas médicas estava associado ao aumento do número de patologias, foi conduzida regressão linear simples.

As análises foram efetuadas por meio do software Statistical Package for the Social Sciences (SPSS) versão 17.0. O nível de significância considerado foi de 0,05 para todas as análises.

\section{Resultados}

Os resultados referentes às prevalências de agravos à saúde e doenças nos últimos 12 meses, segundo sexo, estão descritos na Tabela 1, na qual se observa que as condições mais frequentes foram a hipertensão arterial e a incontinência urinária, relatadas por 37,9\% e 25,1\% dos participantes, respectivamente.

Houve diferença estatisticamente significativa entre os sexos masculino e feminino para patologias do coração $(\mathrm{p}=0,072)$ e tumor/câncer $(\mathrm{p}=0,013)$, mais prevalentes entre os homens, e para agravos como a artrite reumatoide $(\mathrm{p}=0,002)$, a depressão $(\mathrm{p}=0,040)$, a incontinência urinária $(\mathrm{p}=0,037)$ e a osteoporose $(\mathrm{p}=0,000)$, mais prevalentes entre as mulheres. 


\section{Artigo original}

Tabela 1. Prevalência de patologias e agravos à saúde relatados segundo sexo- Estudo FIBRA/JF. Juiz de Fora, MG, 2009 ( $N=426$ )

\begin{tabular}{|c|c|c|c|c|c|c|c|}
\hline \multirow{2}{*}{ Problema de saúde } & \multicolumn{2}{|c|}{ Masculino } & \multicolumn{2}{|c|}{ Feminino } & \multicolumn{2}{|c|}{ Total } & \multirow{2}{*}{ p-valor } \\
\hline & $\mathrm{n}$ & $\%$ & $\mathrm{n}$ & $\%$ & $\mathrm{~N}$ & $\%$ & \\
\hline Hipertensão arterial & 48 & 36,9 & 114 & 38,4 & 162 & 37,9 & 0,775 \\
\hline Diabetes mellitus & 13 & 10,0 & 32 & 10,8 & 45 & 10,5 & 0,810 \\
\hline Doença cardíaca & 16 & 12,3 & 23 & 7,7 & 39 & 9,1 & 0,072 \\
\hline Doença pulmonar & 7 & 5,4 & 15 & 5,1 & 22 & 5,2 & 0,886 \\
\hline Artrite/reumatismo & 14 & 10,8 & 71 & 23,9 & 85 & 19,9 & 0,002 \\
\hline Câncer & 8 & 6,2 & 5 & 1,7 & 13 & 3,0 & 0,013 \\
\hline Osteoporose & 3 & 2,3 & 42 & 14,1 & 45 & 10,5 & 0,000 \\
\hline Incontinência urinária & 24 & 18,5 & 83 & 27,9 & 107 & 25,1 & 0,037 \\
\hline Depressão & 12 & 9,2 & 50 & 16,8 & 62 & 14,5 & 0,040 \\
\hline Derrame/AVC/isquemia & 6 & 4,6 & 8 & 2,7 & 14 & 3,3 & 0,305 \\
\hline Tabagista & 13 & 10,0 & 22 & 7,4 & 35 & 17,4 & 0,208 \\
\hline Fraturas por queda & 03 & 2,3 & 17 & 5,7 & 20 & 4,7 & 0,168 \\
\hline
\end{tabular}

Tabela 2. Prevalência de patologias e agravos à saúde relatados segundo faixa etária - Estudo FIBRA/JF. Juiz de Fora, MG, 2009 ( $N=426$ )

\begin{tabular}{|c|c|c|c|c|c|c|c|}
\hline \multirow{2}{*}{ Problema de saúde } & \multicolumn{2}{|c|}{$65-74$ anos } & \multicolumn{2}{|c|}{$75-84$ anos } & \multicolumn{2}{|c|}{$\geq 85$ anos } & \multirow{2}{*}{$\mathrm{p}$-valor } \\
\hline & $\mathrm{n}$ & $\%$ & $\mathrm{n}$ & $\%$ & $\mathrm{~N}$ & $\%$ & \\
\hline Hipertensão arterial & 90 & 38,3 & 58 & 37,9 & 14 & 35,9 & 0,960 \\
\hline Diabetes mellitus & 24 & 10,2 & 20 & 13,1 & 1 & 2,6 & 0,157 \\
\hline Doença cardíaca & 16 & 6,8 & 18 & 11,8 & 5 & 12,8 & 0,188 \\
\hline Doença pulmonar & 8 & 3,4 & 12 & 7,8 & 2 & 5,1 & 0,154 \\
\hline Artrite/reumatismo & 45 & 19,1 & 33 & 21,6 & 7 & 17,9 & 0,801 \\
\hline Câncer & 8 & 3,4 & 3 & 2,0 & 2 & 5,1 & 0,526 \\
\hline Osteoporose & 18 & 7,7 & 20 & 13,1 & 7 & 17,9 & 0,068 \\
\hline Incontinência urinária & 50 & 21,3 & 44 & 28,8 & 13 & 33,3 & 0,115 \\
\hline Depressão & 32 & 13,6 & 26 & 17,0 & 4 & 10,3 & 0,477 \\
\hline Derrame/AVC/isquemia & 7 & 3,0 & 6 & 3,9 & 1 & 2,6 & 0,849 \\
\hline Tabagismo & 25 & 10,6 & 6 & 3,9 & 4 & 10,3 & 0,112 \\
\hline Fraturas por queda & 4 & 1,7 & 11 & 7,2 & 5 & 12,8 & 0,003 \\
\hline
\end{tabular}


Pricila C. C. Ribeiro e cols. • Perfil clínico e serviços de saúde em idosos

Tabela 3. Acesso a plano de saúde segundo características sociodemográficas - Estudo FIBRA/JF. Juiz de Fora, MG, 2009 $(\mathrm{N}=426)$

\begin{tabular}{|c|c|c|c|c|c|}
\hline & \multicolumn{4}{|c|}{ Possui Plano de Saúde } & \multirow{3}{*}{$p$-valor } \\
\hline & \multicolumn{2}{|c|}{ Sim } & \multicolumn{2}{|c|}{ Não } & \\
\hline & $\mathrm{N}$ & $\%$ & $\mathrm{~N}$ & $\%$ & \\
\hline \multicolumn{6}{|l|}{ Gênero } \\
\hline Masculino & 66 & 25,7 & 63 & 37,3 & \multirow{2}{*}{0,008} \\
\hline Feminino & 191 & 74,3 & 106 & 62,7 & \\
\hline \multicolumn{6}{|l|}{ Faixa Etária } \\
\hline $65-69$ & 64 & 24,9 & 58 & 34,3 & \multirow{3}{*}{0,058} \\
\hline $70-79$ & 125 & 48,6 & 79 & 46,7 & \\
\hline$>80$ & 68 & 26,5 & 32 & 18,9 & \\
\hline \multicolumn{6}{|c|}{ Anos de estudo } \\
\hline 0 & 11 & 4,7 & 26 & 15,9 & \multirow{5}{*}{$<0,001$} \\
\hline $1-3$ & 43 & 18,2 & 59 & 36,0 & \\
\hline $4-7$ & 106 & 44,9 & 63 & 38,4 & \\
\hline $8-11$ & 70 & 29,7 & 14 & 8,6 & \\
\hline$>12$ & 6 & 2,5 & 2 & 1,2 & \\
\hline \multicolumn{6}{|c|}{ Renda em salários mínimos* } \\
\hline 0 a 1 & 78 & 30,4 & 94 & 55,6 & \multirow{3}{*}{$<0,001$} \\
\hline 1 a 3 & 81 & 31,5 & 58 & 34,3 & \\
\hline$>3$ & 98 & 38,1 & 17 & 10,1 & \\
\hline
\end{tabular}

* Salário mínimo equivalente a $\mathrm{R} \$ 465,00$ no período do estudo.

Entre as faixas etárias, observou-se uma prevalência significativamente mais alta de fraturas por quedas para a faixa etária mais velha, comparada às faixas entre 65 e 84 anos ( $p=0,003)$. Para a prevalência dos demais agravos investigados não houve diferença significativa entre as faixas etárias (ver Tabela 2).

Quanto à funcionalidade, $97,4 \%$ da amostra relatou manter-se independente para a realização de ABVD. Para a realização das AIVD, 60,2\% dos respondentes mantinham-se independentes e 39,9\% apresentaram dependência parcial. Foi maior o percentual de mulheres que o de homens com alguma dependência para AIVD (54,1\% contra $27,7 \%$, respectivamente; $\mathrm{p}=0,001)$ e também para ABVD $(3,4 \%$ contra $0,8 \%$, respectivamente; $\mathrm{p}=0,046)$.

A população estudada relatou ter realizado em média 5,61 ( $\mathrm{DP}=4,64)$ consultas médicas nos 12 meses anteriores à pesquisa. Além disto, $12,6 \%$ dos entrevistados relataram ter recebido algum profissional de saúde para atendimento domiciliar; $4,2 \%$ afirmaram não ter demandado atendimento médico neste mesmo período; $1,6 \%$ afirmaram ter necessidade do atendimento, mas optaram por não procurar atendimento médico e $0,5 \%$ alegaram ter dificuldade para conseguir uma consulta médica. Quanto à internação hospitalar, 18,3\% dos participantes ficaram internados por pelo menos uma noite nos 12 meses anteriores à pesquisa. Foi relatada permanência no leito por motivo de doença por $13,1 \%$ dos entrevistados, sendo que destes $76,8 \%(n=43)$ eram mulheres.

A média de medicamentos utilizada pelos participantes foi de 3,59 $(\mathrm{DP}=2,52)$ unidades. Quanto ao acesso à medicação, 48,5\% relataram ter comprado com recursos próprios e $8,7 \%$ conseguiram a medicação em postos de saúde. Além disto, 14,5\% afirmaram ter deixado de tomar a medicação devido à dificuldade financeira para adquirir o que foi prescrito pelo médico.

Dentre os participantes, $60,2 \%$ afirmaram possuir plano de saúde. Destes, $60,3 \%$ relataram ter ido a cinco ou mais consultas médicas no último ano. Este percentual foi significativamente mais alto $(\mathrm{p}=0,01)$ que o observado entre os idosos sem acesso a plano de saúde $(41,4 \%)$. O acesso a plano de saúde foi maior para as mulheres comparado aos homens, para ogrupo com idadesuperior a 80 anos comparado às demais faixas etárias e para os grupos com escolaridade mais alta comparados ao grupo de analfabetos e com baixa escolaridade (ver Tabela 3). Segundo a presença de patologias, houve diferença significativa quanto ao acesso a plano de saúde somente para o relato de osteoporose $(\mathrm{p}=0,002)$.

Quanto à frequência de uso de serviços de saúde, observou-se que o aumento no número de doenças 


\section{Artigo original}

Tabela 4. Número médio de consultas médicas nos últimos 12 meses segundo patologia relatada - Estudo FIBRA/JF. Juiz de Fora, MG, 2009 ( $\mathrm{N}=426)$

\begin{tabular}{|c|c|c|}
\hline Problema de saúde & Média de Consultas (DP) & $\mathrm{p}$-valor \\
\hline \multicolumn{3}{|l|}{ Hipertensão arterial } \\
\hline Sim & $6,01(4,62)$ & \multirow{2}{*}{0,173} \\
\hline Não & $5,37(4,65)$ & \\
\hline \multicolumn{3}{|l|}{ Doença cardíaca } \\
\hline Sim & $8,47(6,45)$ & \multirow{2}{*}{0,000} \\
\hline Não & $5,32(4,34)$ & \\
\hline \multicolumn{3}{|l|}{ Diabético } \\
\hline Sim & $7,20(5,08)$ & \multirow{2}{*}{0,015} \\
\hline Não & $5,42(4,56)$ & \\
\hline \multicolumn{3}{|l|}{ Doença pulmonar } \\
\hline Sim & $9,09(6,53)$ & \multirow{2}{*}{0,000} \\
\hline Não & $5,42(4,45)$ & \\
\hline \multicolumn{3}{|l|}{ Artrite/reumatismo } \\
\hline Sim & $6,29(4,97)$ & \multirow{2}{*}{0,131} \\
\hline Não & $5,44(4,55)$ & \\
\hline \multicolumn{3}{|l|}{ Câncer } \\
\hline Sim & $8,15(4,90)$ & \multirow{2}{*}{0,045} \\
\hline Não & $5,53(4,62)$ & \\
\hline \multicolumn{3}{|l|}{ Derrame/AVC/isquemia } \\
\hline Sim & $9,21(7,14)$ & \multirow{2}{*}{0,003} \\
\hline Não & $5,49(4,50)$ & \\
\hline \multicolumn{3}{|l|}{ Osteoporose } \\
\hline Sim & $7,16(5,37)$ & \multirow{2}{*}{0,018} \\
\hline Não & $5,43(4,52$ & \\
\hline \multicolumn{3}{|l|}{ Incontinência urinária } \\
\hline Sim & $5,75(4,52)$ & \multirow{2}{*}{0,718} \\
\hline Não & $5,57(4,69)$ & \\
\hline \multicolumn{3}{|l|}{ Depressão } \\
\hline Sim & $6,93(4,54)$ & \multirow{2}{*}{0,016} \\
\hline Não & $5,39(4,63)$ & \\
\hline \multicolumn{3}{|l|}{ Fratura decorrente de queda } \\
\hline Sim & $5,25(3,88)$ & \multirow{2}{*}{0,218} \\
\hline Não & $6,82(5,40)$ & \\
\hline
\end{tabular}

crônicas relatadas esteve associado $(\mathrm{p}<0,001)$ a um aumento de $26 \%$ da frequência de consultas médicas realizadas pelos idosos entrevistados. Não houve diferença entre as faixas etárias $(\mathrm{p}=0,766)$, os sexos ( $\mathrm{p}=0,709)$; os grupos de escolaridade ( $\mathrm{p}=0,581$ ), de situação conjugal $(p=0,372)$ nem de renda $(p=0,204)$ quanto ao número de consultas realizadas nos últimos 12 meses. A Tabela 4 descreve o número médio de consultas médicas gerais, segundo o relato de presença de patologia ou agravo da saúde. Pode-se observar que foi maior a média de consultas para os idosos que relataram doença cardíaca $(\mathrm{p}<0,001)$, diabetes $(\mathrm{p}=0,015)$, doença pulmonar ( $p<0,001)$, câncer $(\mathrm{p}=0,045), \mathrm{AVC}(\mathrm{p}=0,003)$, osteoporose
( $\mathrm{p}=0,018$ ) e depressão $(\mathrm{p}=0,016)$ quando comparado àqueles que relataram não ter sido diagnosticados com cada um destes agravos respectivamente.

\section{Discussão}

Na presente investigação foram descritas as prevalências de patologias crônicas e a demanda por assistência à saúde em uma população de idosos residentes na comunidade. O perfil clínico retrata idosos sem grandes prejuízos funcionais para atividades de autocuidado, mesmo sendo acometidos por pelo menos uma doença crônica. Destaca-se que 97,4\% da amostra mantinha-se independente para as ABVD e 
54,1\% para as AIVD. Resultado semelhante foi obtido em outro inquérito populacional realizado com idosos residentes no município de Juiz de Fora, no qual os pesquisadores relataram que $55,6 \%$ dos idosos eram independentes para as AIVD. ${ }^{21}$

A hipertensão arterial destacou-se como a doença crônica mais prevalente $(37,9 \%)$ nesta população, compatível com o que vem sendo relatado na literatura acerca do perfil clínico do idoso brasileiro. ${ }^{21,22}$ Contudo, esta prevalência apresentou-se inferior à identificada para a população geral de Minas Gerais, que foi de $58,3 \%$ segundo o PAD-MG|2011, inclusive para população da Zona da Mata, região onde se localiza o município estudado, cuja prevalência de hipertensão foi $54,9 \%{ }^{23}$ O mesmo aconteceu para a prevalência de diabetes, que foi de $10,5 \%$ no presente estudo contra $18,3 \%$ no estado de Minas Gerais. ${ }^{23}$ Estes resultados podem indicar um desconhecimento da população estudada acerca destes diagnósticos devido à reduzida busca ativa ou ainda apontar uma limitação metodológica, uma vez que as informações sobre estas patologias foram obtidas a partir do autorrelato.

Chrestani, Santos e Matijasevich, em estudo de base populacional com indivíduos a partir de 20 anos, evidenciou que a prevalência de hipertensão obtida por autorrelato foi de $33,6 \%$, na amostra geral do estudo, e de $61,9 \%$ para o grupo com 60 anos ou mais e, quando obtida por aferição objetiva ou por uso de medicação anti-hipertensiva, foi de $29,5 \%$ e $65,4 \%$, respectivamente. ${ }^{24}$ Reforça-se, assim, a hipótese de subestimação da prevalência da hipertensão nos estudos populacionais com idosos, quando utilizado o autorrelato, dada a possibilidade desta patologia estar presente sem a manifestação de sintomas, o que faz com que seu portador permaneça sem o diagnóstico até que se agrave o quadro clínico. Por isto, destaca-se a urgência de intervenções preventivas e diagnósticas do setor de saúde que possam auxiliar no controle e na detecção precoce do problema.

O aumento das estratégias voltadas para a melhora dos hábitos alimentares e a prática de atividade física na população pode auxiliar no controle de patologias como diabetes e de hipertensão., ${ }^{3,7}$ Conforme destacaram Schmidt e colaboradores, quando são implantadas adequadas políticas públicas de saúde, resultados favoráveis podem ser obtidos, como observou-se no caso da diminuição das taxas brasileiras de mortalidade por doenças respiratórias e cardiovasculares no período de 1996 a 2007 em decorrência de estratégias que buscaram a redução do consumo de tabaco e a ampliação da atenção em saúde primária. ${ }^{4}$ A prevalência de outras condições clínicas investigadas no presente estudo apresentou-se bem próxima às descritas para a população mineira, como nos quadros de doenças pulmonares, câncer e artrite/reumatismos. Por outro lado, a prevalência de depressão na população estudada $(14,9 \%)$ foi maior quando comparada aos dados do PAD-MG (10\%). ${ }^{23}$ Não obstante, este resultado foi ainda inferior à prevalência de 19,2\% obtida para transtorno depressivo em outro inquérito realizado com idosos de Juiz de Fora. ${ }^{21}$ A divergência nos resultados sobre a depressão pode ser devido à variabilidade na forma de mensurar o transtorno entre os diversos inquéritos. Cabe ressaltar a necessidade de ampliação dos estudos sobre os sintomas depressivos na população idosa, uma vez que os agravos da saúde mental estão associados a inúmeros desfechos negativos na velhice.

No que se refere ao uso dos serviços de saúde, observou-se uma demanda maior por hospitalização na população estudada $(18,3 \%)$ comparada à população idosa geral no estado de Minas Gerais, na qual 10,8\% dos entrevistados relatou necessidade de internação hospitalar no último ano. A alta frequência de utilização do serviço de internação pode ser explicada pela forma como esta informação foi coletada junto a amostra, com a pergunta: "O senhor precisou ser internado por pelo menos uma noite?". Desta forma, foram mensuradas internações, não só para atenção às demandas por longo período de intervenção hospitalar, mas também para condução de exames e observações clínicas. $\mathrm{O}$ amplo acesso ao serviço privado de assistência à saúde na população estudada corrobora esta hipótese de facilitação do uso do serviço de internação hospitalar. No presente estudo, a adesão a planos de saúde foi superior ao observado na população geral do estado de Minas Gerais (60,2\% contra 26,1\%, respectivamente), superando inclusive o da região metropolitana de Belo Horizonte, que seria de um terço dos idosos, segundo dados do último Boletim PAD-MG|2011. ${ }^{23}$ Este boletim apontou uma grande variabilidade quanto à cobertura de planos de saúde nesta região do país.

No Brasil, a cobertura de assistência das operadoras privadas de saúde vem aumentando significativamente, estando o maior acesso, proporcionalmente, entre as faixas etárias mais velhas e as camadas mais escolarizadas. ${ }^{2,25}$ Compatível com estes dados, encontrou-se no presente estudo maiores percentuais de usuários de plano de saúde nos grupos com maiores níveis de escolaridade e com idade mais avançada. Especificamente na região estudada no presente estudo, 


\section{Artigo original}

Juiz de Fora, ogrande percentual de indivíduos nas faixas etárias mais velhas e as características socioeconômicas favoráveis da população podem explicar o elevado acesso ao serviço privado de assistência à saúde.

Adicionalmente, foi observado maior percentual de mulheres com acesso a plano privado comparado aos homens. Esse aumento do acesso ao plano de saúde entre os mais velhos e as mulheres pode estar associado ao aumento das chances destes grupos serem acometidos por doenças crônicas e incapacitantes e, consequentemente, demandarem com mais frequência tratamento médico. Além disto, é reconhecida a maior frequência de comportamentos de busca por atendimento médico assistencial e preventivo entre as mulheres idosas em comparação aos homens, sendo esta diferença explicada, principalmente, pelo fato de maiores chances de adoecimento para o sexo feminino. ${ }^{26,27}$ Assim, torna-se plausível supor que as famílias se organizem para custear para seus membros mais velhos, principalmente mulheres, o acesso ao setor privado de assistência à saúde, de forma a suprir o que junto ao setor público pode ser menos garantido, como curto tempo de espera para exames e cirurgias. Por um lado, pode-se supor que o grupo com acesso a plano de saúde tenha um perfil de maior prevalência de patologias crônico-degenerativas em decorrência da maior demanda e urgência por assistência à saúde. Por outrolado, pode-se imaginar uma reduzida prevalência de algumas patologias como, por exemplo, as doenças cardiovasculares, que dependem de hábitos de vida saudável mais prováveis neste grupo, resultantes de outros fatores como a maior renda e a alta escolaridade que são observados no grupo de idosos beneficiários de planos de saúde. ${ }^{25}$

No que se refere ao uso do serviço de saúde e acesso a plano de saúde, são controversos os achados sobre a demanda por internação hospitalar. No estudo de Louvison e colaboradores, com idosos do Estudo SABE, conduzido no município de São Paulo, a frequência de internações hospitalares foi maior entre os assistidos no setor público. ${ }^{28}$ Ao contrário, Barros, Francisco, Zanchetta et al apresentaram maior frequência de internação entre indivíduos filiados a planos de saúde comparado aos não filiados. ${ }^{7}$ No presente estudo, não foram observadas diferenças quanto à necessidade de internação e o acesso a plano de saúde.

Os achados sobre a realização de consultas médicas parecem consensuais na literatura, indicando maior realização de consultas por parte da população que tem plano de saúde. No presente estudo, por exemplo, foi maior a frequência de realização de mais de quatro consultas nos últimos 12 meses entre os idosos com acesso a plano de saúde comparado ao grupo sem plano de saúde. O aumento do número de patologias crônicas também esteve associado ao aumento do número de consultas médicas. Estes dados corroboram o que vem sendo apresentado na literatura sobre aumento do uso de serviço de saúde em função da presença de doenças. ${ }^{7,26,29}$ Contudo, deve-se considerar que não só a presença de múltiplas condições crônicas, mas também a forma como o setor de saúde oferece o serviço pode influenciar na quantidade de consultas realizadas. Enquanto no setor privado o usuário pode procurar de forma independente a consulta de especialistas, por exemplo, no setor público este tipo de consulta depende de encaminhamentos e podem implicar em maior tempo de espera entre as consultas médicas realizadas em um ano.

Cabe destacar que as conclusões do presente estudo são limitadas quanto à informação se o uso do serviço de saúde, seja consulta ou internação, foi realizado junto ao setor público ou privado. Sabe-se que no Brasil existe uma interface, com uso do setor público e privado, dos serviços de cuidado com a saúde de forma que as intervenções de maior custo, como por exemplo cirurgias e hospitalização de longa duração, podem ser feitas no setor público mesmo entre os usuários de consultas médicas via plano de saúde ou atendimento médico autônomo do setor privado. ${ }^{30-32}$

De forma geral, o presente estudo mostrou que o uso mais frequente dos serviços de saúde, principalmente consultas médicas, não estava relacionado às características demográficas, como aumento da idade e da escolaridade, mas esteve associado à renda, ao acesso a plano de saúde e à presença de comorbidades. A população investigada representa idosos saudáveis cujos principais acometimentos de saúde são doenças crônicas controláveis e não incapacitantes. Esse perfil é condizente com o da população idosa brasileira, que se mantém, em sua maioria, capaz de realizar suas atividades de vida diária, e trata-se de idosos sem graves prejuízos cognitivos e de mobilidade. Assim, as demandas por serviços de assistência à saúde apresentadas neste trabalho devem ser pensadas enquanto reflexos do perfil clínico mencionado, podendo se diferenciar, substancialmente, de contextos que focalizam grupos de idosos com graves comprometimentos. Fialho, Lima-Costa, Giacomin e colaboradores, por exemplo, em inquérito com idosos residentes na região Metropolitana de Belo Horizonte/MG, mostraram que é maior o uso de serviços de 
saúde, sobretudo os mais onerosos, na população mais velha acometida por incapacidade funcional. ${ }^{33}$ Estes autores ressaltaram que entre os critérios de estruturação da oferta de serviços de saúde devem estar os prejuízos funcionais mais do que a presença de doenças.

Futuros estudos devem ampliar a descrição do acesso à assistência para o cuidado com a saúde entre idosos, sejam no setor público ou privado, bem como a investigação de como as estratégias preventivas e assistenciais podem reduzir as prevalências das doenças crônicas e aumentar as chances de envelhecimento saudável na população.

\section{Financiamento}

Conselho Nacional dePesquisa-CNPq(No.555087/20069) e Fundação de Amparo à Pesquisa do Estado de Minas Gerais - FAPEMIG (CHE - APQ-01145-14)

\section{Referências}

1. Duarte EC, Barreto SM. Transição demográfica e epidemiológica: a Epidemiologia e Serviços de Saúde revisita e atualiza o tema. Epidemiol. Serv. Saúde. 2012;21(4):529-532.

2. Instituto Brasileiro de Geografia e Estatística. Um panorama da saúde no Brasil: acesso e utilização dos serviços, condições de saúde e fatores de risco e proteção à saúde: 2008. Rio de Janeiro:IBGE; 2010.

3. Malta DC, Moura L, Prado RR, et al. Mortalidade por doenças crônicas não transmissíveis no Brasil e suas regiões, 2000 a 2011. Epidemiol Serv Saúde. 2014;23(4):599-608.

4. Schmidt Ml; Duncan BB, Silva GA, et al. Chronic non-communicable diseases in Brazil: burden and current challenges. Lancet. 2011;377(9781):1949-1961.

5. Brandão CMR, Guerra Júnior AA, Cherchiglia ML, et al. Gastos do Ministério da Saúde do Brasil com medicamentos de alto custo: uma análise centrada no paciente. Value in Health. 2011;14:S71-S77.

6. Da Silveira RE, Santos AS, Sousa MC, et al. Gastos relacionados a hospitalizações de idosos no Brasil: perspectivas de uma década. Einstein. 2013;11(4):514-520.

7. Barros MBA, Francisco PMSB, Zanchetta LM, et al. Tendências das desigualdades sociais e demográficas na prevalência de doenças crônicas no Brasil, PNAD: 2003-2008. Ciênc Saúde Coletiva. 2011;16(9):3755-3768.

8. Pinto JM, Neri AL. Doenças crônicas, capacidade funcional, envolvimento social e satisfação em idosos comunitários: Estudo Fibra. Ciênc Saúde Coletiva. 2013;18(12):3449-3460.

9. Campos ACV, Almeida MHM, Campos GV, et al. Prevalência de incapacidade funcional por gênero em idosos brasileiros: uma revisão sistemática com metanálise. Rev Bras Geriatr Gerontol. 2016;19(3):545-559.

10. Rodrigues NO, Neri AL. Vulnerabilidade social, individual e programática em idosos da comunidade: dados do estudo FIBRA, Campinas, SP, Brasil. Ciênc Saúde Coletiva. 2012;17(8):21292139.

11. Pimenta FB, Pinho L, Silveira MF, et al. Fatores associados a doenças crônicas em idosos atendidos pela Estratégia de Saúde da Família. Ciênc Saúde Coletiva. 2015;20(8):2489-2498.
12. Gronlund CJ, Berrocal VJ, White-Newsome JL, et al. Vulnerability to extreme heat by socio-demographic characteristics and area green space among the elderly in Michigan, 1990-2007. Environ res. 2015;136:449-461.

13. Organização das Nações Unidas. Political declaration of the high-level meeting of the general assembly on the prevention and control of non-communicable diseases. In: High-level Plenary Meeting of the General Assembly [Internet]. New York: United Nations; 2011 [acesso em 09 abr 2018]. Disponível em: http:// www.un.org/ga/search/view_doc.asp?symbol=A/66/L.1

14. Ministério da Saúde. Plano de ações estratégicas para o enfrentamento das doenças crônicas não transmissíveis (DCNT) no Brasil 2011-2022. Brasília: Ministério da Saúde; 2011.

15. Lourenço RA, Moreira VLG, Banhato EFC, et al. Prevalence of frailty and associated factors in a community-dwelling older people cohort living in Juiz de Fora, Minas Gerais, Brazil: Fibra-JF Study. Ciênc Saúde Coletiva. 2019;24(1):35-44.

16. Roberto AL, Mármora CHC, Banhato EFC, et al. FIBRA Juiz de Fora: História e Fundamentos Metodológicos. Revista Hospital Universitário Pedro Ernesto; 2018.

17. Katz S, Ford AB, Moskowitz RW, et al. Studies of illness in the aged. The index of ADL: a standardized measure of biological and psychosocial function. JAMA. 1963;185:94-99.

18. Lino VTS, Pereira SRB, Camacho LAB, et al. Adaptação transcultural da Escala de Independência em Atividades da Vida Diária (Escala de Katz). Cad Saúde Pública. 2008;24(1):103-112.

19. Lawton MP, Brody EM. Assessment of older people: self-maintaining and instrumental activities of daily living. Gerontologist. 1969;9(3):179-186.

20. Santos RL, Virtuoso Júnior JS. Confiabilidade da versão brasileira da escala de atividades instrumentais da vida diária. RBPS. 2008;21(4):290-296.

21. Cruz DT, Vieira MT, Bastos RR, et al. Fatores associados à fragilidade em uma população de idosos da comunidade. Rev Saúde Pública. 2017;51:1-13.

22. Malta DC, Bernal RTI, Andrade SSCA, et al. Prevalência e fatores associados com hipertensão arterial autorreferida em adultos brasileiros. Rev Saúde Pública. 2017;51:1-11s.

23. Minas Gerais. Boletim PAD MG | 2011: perfil da população idosa de Minas Gerais. Belo Horizonte: Fundação João Pinheiro; 2014.

24. Chrestani MAD, Santos IS, Matijasevich AM. Hipertensão arterial sistêmica auto-referida: validação diagnóstica em estudo de base populacional. Cad Saúde Pública. 2009;25(11):2395-2406.

25. Malta DC, Moura EC, Oliveira M, et al. Health insurance users: self-reported morbidity and access to preventive tests according to a telephone survey, Brazil, 2008. Cad Saúde Pública. 2011;27(1):57-66.

26. De Almeida AN. O acesso aos serviços de saúde pelos idosos no Brasil com base na Pesquisa Nacional por Amostra de Domicílios (PNAD) entre 1998 e 2008. J Bras Econ Saúde. 2015;7(1):43-52.

27. Borim FSA, Guariento ME, Almeida EA. Perfil de adultos e idosos hipertensos em unidade básica de saúde. Rev Soc Bras Clín Méd. 2011;9(2):107-11.

28. Louvison MCP, Lebrão ML, Duarte YAO, et al. Inequalities in access to health care services and utilization for the elderly in São Paulo, Brazil. Rev Saúde Pública. 2008;42(4):733-740.

29. Barros MBA, Cesar CLG, Carandina L, et al. Desigualdades sociais na prevalência de doenças crônicas no Brasil, PNAD-2003. Ciênc Saúde Coletiva. 2006;11(4):911-926.

30. Santos IS, Ugá MAD, Porto SM. O mix público-privado no Sistema de Saúde Brasileiro: financiamento, oferta e utilização de 


\section{Artigo original}

serviços de saúde. Ciênc Saúde Coletiva. 2008;13(5):1431-1440. 31. Porto SM, Santos IS, Ugá MAD. A utilização de serviços de saúde por sistema de financiamento. Ciênc Saúde Coletiva. 2006;11(4):895-910.

32. Bahia L, Simmer E, Oliveira DC. Cobertura de planos privados de saúde e doenças crônicas: notas sobre utilização de procedi- mentos de alto custo. Ciênc Saúde Coletiva. 2004;9(4):921-929. 33. Fialho CB, Lima-Costa MF, Giacomin KC, et al. Capacidade funcional e uso de serviços de saúde por idosos da Região Metropolitana de Belo Horizonte, Minas Gerais, Brasil: um estudo de base populacional. Cad Saúde Pública. 2014;30(3):599-610. 